\title{
A Mathematical Model for Prediction the Embedment Depth of the Contiguous Piles Used in the Interchange of Zakho Entrance
}

\author{
Najdat S. Akrawi*, Shimal A. Ahmed \\ Department of Civil Engineering, College of Engineering, University of Duhok, Duhok, Iraq
}

\author{
${ }^{*}$ Corresponding author: \\ Najdat S. Akrawi, \\ Department of Civil \\ Engineering, College of \\ Engineering, University of \\ Duhok, Duhok, Iraq. \\ E-mail: Najdat.sabri@uod.ac \\ Received: 16 October 2019 \\ Accepted: 04 December 2019 \\ Published: 01 December 2019

\section{DOI} \\ 10.25156/pj.v9n2y2019.pp119-124
}

\section{A B S T R A C T}

Determination of the depths of the embedment of contiguous piles requires extensive soil investigation to obtain the soil physical parameters. In addition, a large number of such piles involved in restricted access projects make that depth an essential problem. A simple mathematical model for predicting the depth of embedment using the height of the retained soil, the standard penetration test values, and the bulk unit weight of the soils encountered for 261 pile data sets was introduced using an artificial neural network approach. The coefficient of determination equals to 0.99 for the tested the data reveal that the depth of embedment was accurate against those achieved in Zakho interchange. The importance and parametric studies obtained show that the major parameter which affects the depth of embedment was the height of the retained soil whereas the effect of other parameters is relatively less.

Keywords: Artificial neural network; Contiguous piles; Embedment depth

\section{INTRODUCTION}

Retaining structures using closely bored piles are called contiguous piles. Contiguous piles have many advantages in the aspect of the construction of a retaining wall (Godavarthi et al, 2011). The method of construction involves the creation of a line of deep bores that are then filled with concrete. The soil from either side of the piles could then be excavated to the required geometrical and geotechnical design grades. A suitable capping beam should be placed on the top of the piles for uniformity and maintaining the grades; nevertheless, the piles will not vertically have loaded as for shoring piles. Contiguous piles may be loaded as in many interchanges and intersections that necessarily comprises cut and cover by bridge girders.

The interchange of Zakho entrance project in Dohuk Governorate is an example of selecting such a method of piling as retaining walls to support the excavations for the underpasses that considered an essential part of the project. The advantage of this method of construction is that it is possible to support the adjacent structure before bulk excavation takes place. Contiguous piles are particularly suited to sites with restricted accesses such as structures vicinity of excavations, dense traffic, underground obstructions, and utilities that have made the excavations for conventional retaining wall a difficult task to execute (Kumar, 2008).
The arrangement of the wall either by a line or a curve of piles typically installed at centers $150 \mathrm{~mm}-200 \mathrm{~mm}$ greater than their diameter using continuous flight auger. This means that there are gaps between the piles and therefore contiguous piles are suitable only where the groundwater table is permanently below excavation level (Guo, 2003).

Because of the form of construction, the exposed piles will be fairly rough in appearance. Thus, in most cases, an inner wall that may not be considered as a structural element will be provided, such as sprayed concrete, cladding panels, or modular blocks. A method of drainage for the potential externally exposed water will generally be required between the piles and any inner wall (Chou and $\mathrm{Ou}, 1999)$.

The design of such piles comprising geotechnical and structural issues. The former encounters the depth of embedment the later focus on the moment that exerted to design the pile.

The depth of embedment of the shoring contiguous piles depends primarily on the height that is to be retained, soil type and soil strength, and bulk density whether the piles will be concentrically loaded or not (Puller, 2003).

When the height of the earth to be retained by piles is relatively small, the piling acts as a cantilever wall. The forces acting on the pile wall include: 
1. The active earth pressure on the back of the wall which tries to push the wall away from the backfill

2. The passive pressure in front of the wall below the dredge line. The passive pressure resists the movements of the wall.

The active and passive pressure distributions on the wall are assumed hydrostatic. In the design of the wall, although the Coulomb approach that considers the wall friction tends to be more realistic. Finno, 1989, stated that Rankin's approach (with the angle of wall friction $\delta=0$ ) is normally used for contiguous piles.

In the present study, a mathematical models is developed based on Feed Forward Back Propagation Artificial Neural Network (FFBP-ANN) to determine the depth of embedment (D) of the contiguous piles using the geotechnical data represented by the soil investigation report conducted for the site of Zakho interchange project of 261 piles data set comprising the parameters that represent the height of excavation $(\mathrm{H})$, the adopted standard penetration $n$-values (S), and the bulk density $(\gamma)$ of the retained soil. Importance analysis and Pearson's product-moment correlation coefficient are obtained for the model to assign the impact the parameters involved in the study.

\section{ANN}

ANN, which is an information processing system, makes a simulation of the functions and structure of the human brain (Goh, 1995). This network is a highly interconnected structure consisting of a large number of simple processing elements (i.e., neurons). This network is capable of recognizing similarities when they are presented with new input parameters after appropriately predicting the output pattern, as stated by Liua et al., 2002.

Neural network is applicable as an alternative for some statistical analysis techniques such as multivariable regression, autocorrelation, trigonometric, linear regression, and so on. Using three fundamental components, a network can be comprising the network architecture, transfer function, and learning laws (Simon, 1999).

Several algorithms have been designed to train the neural networks, among which the back-propagation (BP) algorithm is known as the most robust technique (Vanluchene and Sun, 1990). BP can solve predictive complex engineering problems. It makes BP popular among all algorithms for training ANN (Alkroosh and Nikraz, 2012). In general, the feed-forward BP neural network contains at least three layers, namely, input, hidden, and output layers. In each layer, there are some elementary processing units (i.e., neurons) each of which is linked with the next layer through weights. Depending on the given problem, the number of hidden layers and their neurons (nodes) change. The number of input and output neurons is similar to the variables of input and output, respectively. For the purpose of differentiating between different processing units, the values that are named biases are presented in transfer functions. The net output of a neuron or layer to its real or actual output is planned by transfer functions (Shahin et al., 2009). In training the network, the data processing from the input layer to the next (hidden layer) continues until the data reaches the last (output) layer (forward pass). Within this layer, a comparison is performed between the output and the actual values. Then, the difference between output and actual values is propagated back through the network (backward pass), which updates the biases of the individual neurons and the individual weights of the connections.

The process continues until the network error is converged to a threshold that is defined by a corresponding function, which is generally the root mean squared error.

\section{NEURAL ARCHITECTURAL DESIGN AND TRAINING}

The processes that comprising the number of neurons in the hidden layer, the selection the appropriate activation functions and dividing the data to subgroups for training and testing is optimized depending on trial and error to best fit the results between the depth of embedment of the contiguous piles (D) from the ANN results and the corresponding field data used.

Different training functions are available in MATLAB (Howard and Mark, 2002). Levenberg-Marquardt technique has been found efficient in the training process and therefore used to construct the ANN model. This training function is one of the conjugate gradient algorithms that start the training by searching in the steepest descent direction on the first iteration.

Two neurons in the hidden layer were found to be satisfactory to represent the neural network, which turns the network configuration, as shown in Figure 1.

As the input vector, as the control parameters $x_{i}$ are assigned to the input layer, it can determine the input for the hidden layer $I_{j}^{H}$ by

$$
I_{j}^{H}=\theta_{j}+\sum_{i=1}^{N I} w_{j i} x_{i} .
$$




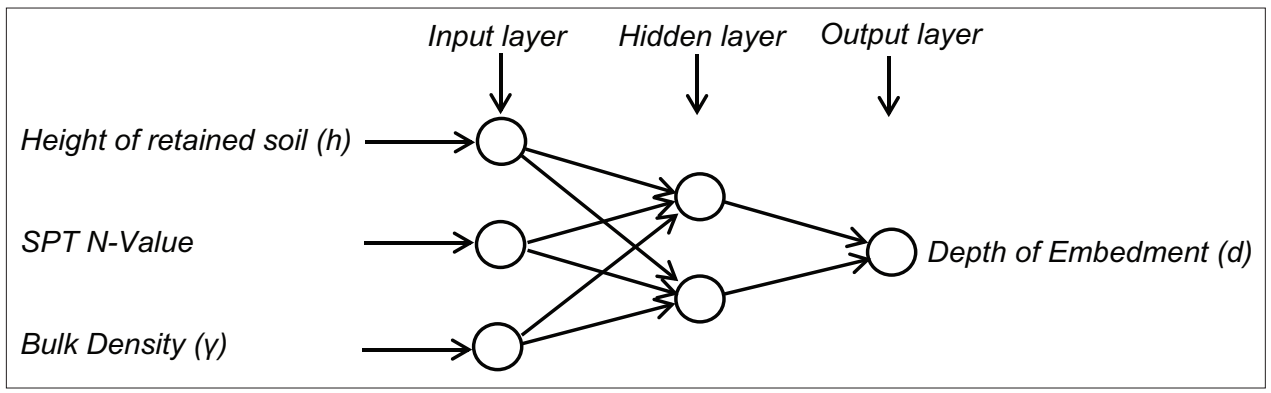

Figure 1: Architecture of the NN model

Where, $\theta_{j}$ is the hidden threshold or bias, $N I$ is the number of the input neurons, and $w_{j i}$ is the synaptic weight that interrelates input neuron $i$ to hidden neuron $j$.

$\mathrm{NH}$ is the number of the neurons in the hidden layer, each neuron of the hidden layer gets its input $I_{j}^{H}$, that to be used as the argument for an activation function $f$, to produce an output $y_{j}^{H}$ and expressed as

$$
y_{j}^{H}=f\left(I_{j}^{H}\right) .
$$

The input for the neurons of the output layer $I_{k}^{O}$ is calculated as

$$
I_{k}^{O}=\theta_{k}+\sum_{i=1}^{N H} w_{k j} y_{j}^{H}
$$

Consequently, $\theta_{k}$ is the output threshold or bias, $N H$ is the number of the input neurons, and $w_{k j}$ is the synaptic weight that interrelates hidden neuron $j$ to output neuron $k$.

And the final network output $y_{k}$ is calculated by an activation function $f$

$$
y_{k}=f\left(I_{k}^{O}\right) .
$$

Different activation functions (i.e., radial, linear, hyperbolic tangent sigmoid, and logarithmic sigmoid) are available and can be used to transfer the input to output data within the hidden layer and the output layer. In the current research, the hyperbolic tangent sigmoid function (TANSIG) was found to be suited between the input and the hidden layer, while the logarithmic sigmoid was found to be used to transfer data between the hidden layer and output layer, as follows:

$\operatorname{TANSIG}(f)=\frac{2}{1+e^{-2 I_{J}^{H}}}-1$, and $\operatorname{LOGSIG}(f)=\frac{1}{1+e^{-I_{k}^{O}}}$.

Before subjecting the input and output data to the network process, it should be normalized to scale-up the inputs and output so that they fall in the range of the selected activation function corresponding to the highest and lowest values, respectively. The obtained normalized output is then need to be de-normalized to the real values, the normalization formula is

$$
Y_{\text {norm }}=\frac{\left(Y_{\text {max }}-Y_{\text {min }}\right) *\left(X_{\text {actual }}-X_{\text {min }}\right)}{X_{\text {max }}-X_{\text {min }}}+Y_{\text {min }}
$$

Where, $Y_{\text {norm }}$ is the normalized value of $X_{\text {actual }}$ The values of $Y_{\max }$ and $Y_{\min }$ are $(-1$ and +1$)$ for the input data and $(0$ and +1$)$ for the output data, respectively. $X_{a c t u a p} X_{m i n,}$ and $X_{\max }$ are the actual, minimum and maximum values of the independent parameters of interest. The same equation above is used for the calculation of the actual depth of embedment (the de-normalization).

The multilayer feed-forward BP technique is subjected to the 261 set of input data set that represents the height of excavation $(\mathrm{H})$, the adopted standard penetration values $(\mathrm{S})$, and the bulk density $(\gamma)$ of the retained soil, as well as, the corresponding set of the targeted output data that represent the depth of embedment (D). The datasets were divided into two subgroups. The first group was 235 data subset is used for training the network and the second group 26 data subset was to be used for testing the network.

Tables 1 and 2 represent the range of the input datasets and the range of the output while the scheme representation of the developed network is shown in Figure 1. The two hidden neurons selected for the FFBP in the network of this study is agreed with the number proposed by Ripley, 1993; Wang, 1994, and Masters, 1994.

$\begin{aligned} & \text { Table 1: Range of the input dataset for the three controlling } \\
& \text { parameters }\end{aligned}$
\begin{tabular}{|lccc}
\hline $\begin{array}{l}\text { Input } \\
\text { parameters }\end{array}$ & $\begin{array}{c}\text { Height of } \\
\text { excavation }(H)\end{array}$ & $n$-value (s) & Bulk density $(\gamma) \gamma)$ \\
\hline Max. & 6.07 & 46 & 20.1 \\
Min. & 2.0 & 29 & 16.9 \\
\hline
\end{tabular}

Table 2: The range of the output parameter Output parameters Depth of embedment (D) $(\gamma)(\gamma)$

\begin{tabular}{ll}
\hline Max. & 4.02 \\
Min. & 0.82
\end{tabular}


As mentioned earlier, the data represent the input and output parameter that processed by ANN should be normalized according to the nature of the activation function selected, the normalization using equation (1) for the input parameters for the range between $(-1$ and +1$)$ subscripted by (n) is as follows:

$$
\begin{gathered}
H_{n}=0.4914 H-1.982 \\
S_{n}=0.1176 S-4.4117 \\
\gamma_{n}=0.625 \gamma-11.5625 .
\end{gathered}
$$

The normalization of the output parameters is also found using the same equation for the range between $(0$ and +1$)$ subscripted by (n) is as follows:

$$
D_{n}=0.3125 D-0.25625
$$

A regression analysis was performed that relate the network response and the corresponding targets, and a correlation coefficient was found. This considered as a measure of how is the output is deviated by the targets.

Figure 2 shows the plot of the depth of embedment (D) required for the contiguous piles obtained by ANN with the corresponding to those achieved in the field. A linear correlation can be observed, and the correlation coefficients are 0.99 for both the training and the test data. Therefore, it is obtained that the ANN model predicts accurately the depth of embedment of the contiguous piles in Zakho intersection against the given input control parameters used.

\section{IMPORTANCE OF INPUT PARAMETERS}

The relative effect or importance of each input parameter into the output parameter in BP-NN process may not be easily formed in the matrix scheme. In this study, such importance is obtained by the method of partitioning weights proposed by Garson, 1991, and adopted by Goh, 1995 , is utilized. The method simply divides the relative weight of individual parameters by the sum of weights of all the input parameters encountered in a specific problem. Figure 3 shows the results obtained for the importance of the input parameters $(\mathrm{H}, \mathrm{S}$, and $\gamma)$. It is obvious from the figure that major important parameter which some 58.15 that influences the depth of embedment of contiguous piles in Zakho intersection was the height of the retained soil $(\mathrm{H})$, this may be consistent to the great effect of the height on the equation proposed by Rankin and coulomb lateral earth pressure theory in comparison with the soil strength represented by the standard penetration value and the bulk density which sum 27.22 and 14.63, respectively.

\section{FORMULATION OF THE MATHEMATICAL MODEL}

The results from BP-ANN of the weights and thresholds values are demonstrated in Tables 3 and 4 . These values can be utilized to formulate the mathematical model expressing

Table 3: The results of the weights and thresholds from ANN of input to the hidden layer

\begin{tabular}{lcccc}
\hline Nodes & \multicolumn{3}{c}{$w_{i j}$} & Hidden threshold $\theta_{j}$ \\
\cline { 2 - 4 } & $\mathrm{l}=1$ & $\mathrm{l}=2$ & $\mathrm{l}=3$ & \\
\hline$j=1$ & 1.4 & -0.7576 & 0.3379 & -2.007 \\
$j=2$ & 0.829 & 0.3493 & 0.213 & 1.0036 \\
\hline
\end{tabular}

ANN: Artificial neural network

Table 4: The weights and output thresholds of hidden to the output layer

\begin{tabular}{lccc}
\hline Nodes & \multicolumn{2}{c}{$w_{i k}$} & Output threshold $\theta_{k}$ \\
\cline { 2 - 3 } & $j=4$ & $j=5$ & \\
\hline$k=3$ & 3.421 & 3.921 & -0.3044 \\
\hline
\end{tabular}

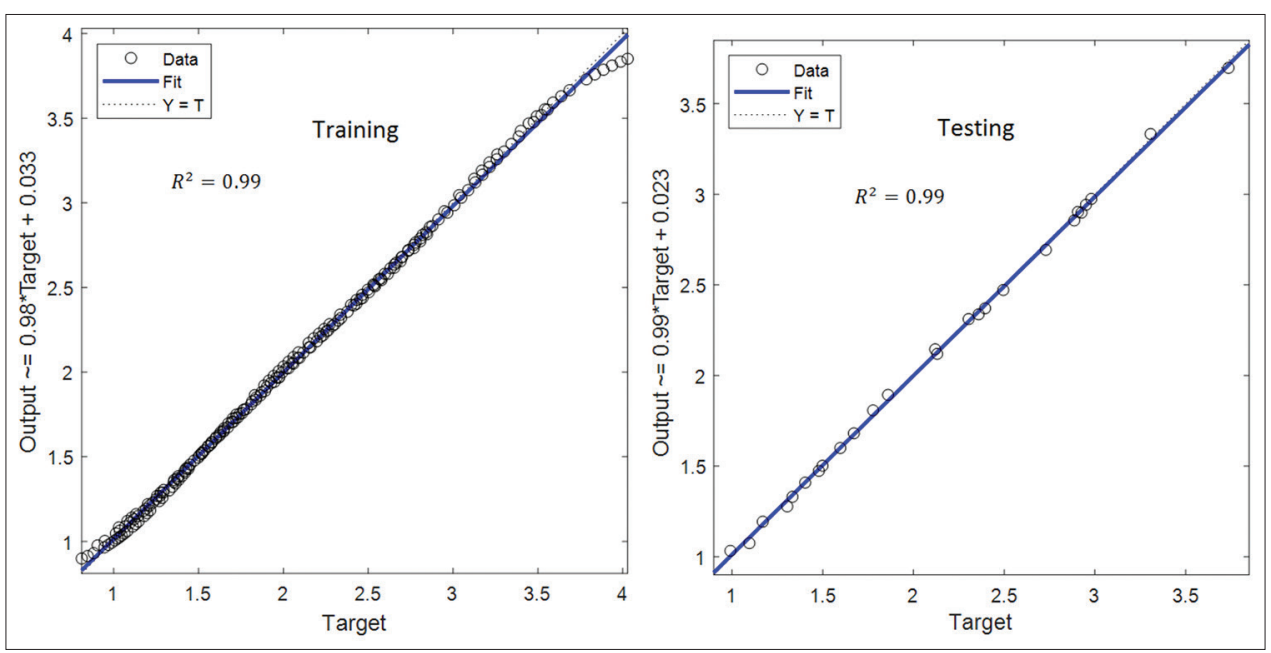

Figure 2: The field and corresponding predicted artificial neural network depth of embedment for the training and testing data 
the embedment depth of the contiguous piles versus the input parameters.

Table 3 lists the results of the weights of the input layer to the hidden layer and the threshold of the hidden layer, while Table 4 lists the results of the weights of the hidden layer to the output layer and the threshold of the output layer.

The mathematic model from the results of the above table can be written as:

$$
D_{n}=\frac{1}{1+e^{-\left[\theta_{3}+\left\{\frac{2 w_{43}}{1+e^{-2 x 1}}\right\}+\left\{\frac{2 w_{53}}{1+e^{-2 \times 2}}\right\}-2\right]}}
$$

where:

$$
\begin{aligned}
& x_{1}=\theta_{1}+w_{11} \cdot H_{n}+w_{21} \cdot S_{n}+w_{31} \cdot \gamma_{n} \\
& x_{2}=\theta_{2}+w_{12} \cdot H_{n}+w_{22} \cdot S_{n}+w_{32} \cdot \gamma_{n}
\end{aligned}
$$

By applying equation (2), the depth of embedment as a mathematical expression can be de-normalized as:

$$
D=0.82+\frac{3.2}{1+e^{\left[2.3044-\left\{\frac{6.842}{1+e^{-2 \times 1}}\right\}\left\{\left\{\frac{7.842}{1+e^{-2 \times 2}}\right\}\right.\right.} .} .
$$

\section{PARAMETRIC RESULTS}

The mathematical model representing the depth of the embedment of contiguous piles in Zakho intersection with the height of excavation, the standard penetration value and the bulk unit weight of the retained soil can be easily utilized by varying one of the control parameters and leaving the other parameters as constant values. This can demonstrate the physical behavior of the model.

Figure 4 shows that, for specific bulk unit weight of the retained soil, the effect of varying values of the (standard penetration test [SPT]) values on the depth of embedment of the contiguous piles, the figure clarify that generally, as the (SPT) values increases the depth of embedment decreases and that the difference becomes more obvious for the greater height of soil retained. This normally attributed to that the thrust of the retained soil becomes lesser as the soil strength increases.

Figure 5 demonstrates, for specific standard penetration value, that as the bulk unit weight increases the depth of embedment (D) increases while the impact of the bulk unit weight $(\gamma)$ is less than the standard penetration value. This can be explained by the fact of the theories of the lateral earth pressure of Rankine's and Coulomb which state that the active thrust varies linearly with the bulk density of the soil retained in contrast the square powered effect of the

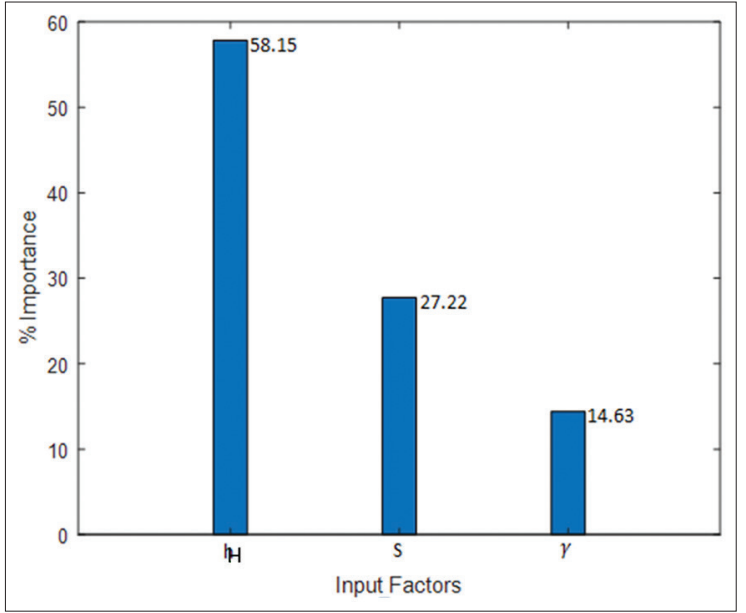

Figure 3: Importance of $\mathrm{H}, \mathrm{S}$, and $\gamma$ of the artificial neural network model

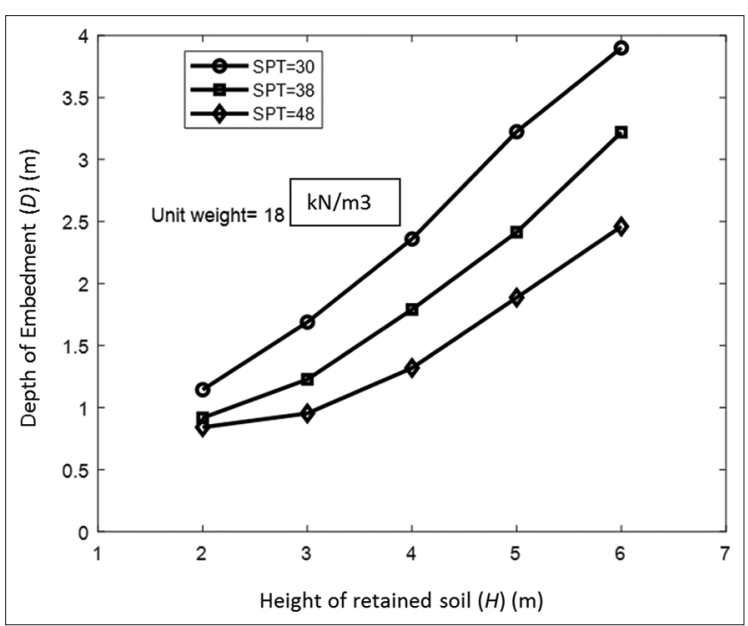

Figure 4: Effect of varying (standard penetration test) values on the depth of the embedment (D) of the studied contiguous piles

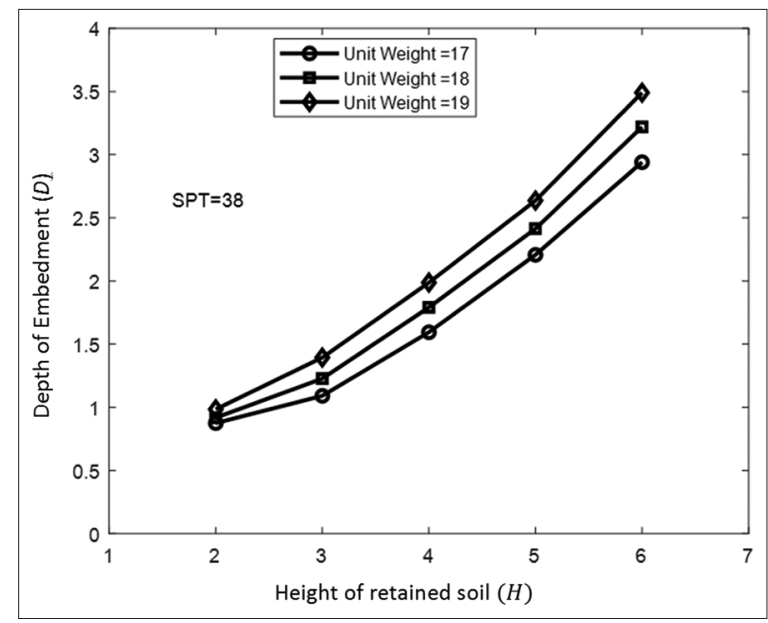

Figure 5: Effect of varying $(\gamma)$ values on the depth of the embedment (D) of the studied contiguous piles

height of the retained soil and the nonlinear effect of the soil strength. 


\section{CONCLUSIONS}

A mathematical model using ANN was introduced for predicting the depth embedment of contiguous piles using geometrical properties of the contiguous piles and the properties of the soil retained for Zakho entrance. ANN approach for modeling was found to be efficient for such purpose. Both the importance and the parametric studies were found that the effect of the standard penetration test is much effective in the determination of the embedment depth than the unit weight, while the most effective parameter was the height of the retained soil. The simple soil parameters involved in the formulating the mathematical model in this study may prompt the engineers to validate the use of other projects.

\section{REFERENCES}

Alkroosh, I. and H. Nikraz. 2012. Predicting axial capacity of driven piles in cohesive soils using intelligent computing. Eng. Appl. Artif. Intell. 25(3): 618-627.

Chou, H. L. and C. Y. Ou. 1999. Boiling failure and resumption of deep excavation. J. Perform. Construct. Facil. ASCE. 13(3): 114-120.

Finno, R. J., D. K. Atmatzidis and S. B. Perkins. 1989. Observed performance of a deep excavation in clay. J. Geotech. Eng. 115(8): 1045-1064.

Garson, G. D. Interpreting neural network connection weights. Artif. Intell. 6(1): 47-51.

Godavarthi, V. R., D. Mallavalli, R. Peddi, N. Katragadda and P. Mulpuru. 2011. Contiguous pile wall as a deep excavation supporting system. Leonardo Electron. J. Pract. Technol.
19: $144-160$.

Goh, A. 1995. Back-propagation neural networks for modeling complex systems. Artif. Intell. Eng. 9(1): 143-151.

Guo, W. D. 2003. A Simplified Approach for Piles Due to Soil Movement. Proceedings of $12^{\text {th }}$ Pan American Conference on Soil Mechanics and Geotechnical Engineering, Cambridge, MIT, USA. p215-2220.

Howard, D. and B. Mark. 2002. Neural Network Toolbox for use with MATLAB, User's Guide, Version 4. The Math Works, Inc., Natick, Massachusetts, United States.

Kumar, M. 2008. Deep Support Systems Using Diaphragm Walls and Contiguous Piles, National Seminar on Deep Excavation in Urban Environment, Mumbai. p1-18.

Liua, S. W., H. H. Jin, J. C. Sungb and C. C. Leeb. 2002. Detection of cracks using neural networks and computational mechanics. Comput. Methods Appl. Mech. Eng. 191(1): 2831-2845.

Masters, T. 1994. Practical Neural Network Recipes in C++. Academic Press, Boston MA.

Puller, M. 2003. Deep Excavations: A Practical Manual. Thomas Telford Ltd., London, England.

Ripley, B. D. 1993. Statistical aspects of neural networks. In: BarndoffNeilsen, O. E., J. I. Jensen and W. S. Kendall. editors. Networks and Chaos-statistical and Probabilistic Aspects. Chapman and Hall, London. p40-123.

Shahin, M. A., M. B. Jaksa and H. R. Maier. 2009. Recent advances and future challenges for artificial neural systems in geotechnical engineering applications. Adv. Artif. Neural Syst. 2009: 308239.

Simon, H. 1999. Neural Networks a Comprehensive Foundation. $2^{\text {nd }}$ ed. Prentice Hall, New Jersey.

Vanluchene, R. D. and R. Sun. 1990. Neural networks in structural engineering. Microcompterin Civil Eng. 5(1): 207-215.

Wang, C. 1994. A Theory of Generalization in Learning Machines with Neural Application. Ph.D Thesis, The University of Pennsylvania, USA. 